\title{
Future directions for research on information technology and educational management
}

\author{
Adrie J. Visscher \\ University of Twente \\ The Netherlands \\ Phil Wild \\ Loughborough University \\ United Kingdom
}

\begin{abstract}
In this paper the features of research on Information Technology and Educational Management (ITEM), as it has been carried out until now, are portrayed. Proposals for future research in terms of research topics and methodology are formulated. In addition the so-called User Acceptance Audit methodology is presented which identifies barriers and promoters to system success.
\end{abstract}

Main conference themes: information technology

Educational areas:

Study topics:

Secondary keywords: administration, management, research 


\section{INTRODUCTION}

The Jerusalem 1994 conference on Information Technology and Educational Management ITEM [1] has shown that the importance of ITEM is increasing strongly world wide. In a number of countries, e.g. Australia, Israel and Hong Kong, enormous sums of money are being invested to let schools and educational administrative bodies benefit from the advantages of computerized information systems (IS). However, in other countries computerized school administration and management is still restricted to some form of office automation.

ITEM has become a new valuable area of research which can provide us with insight into the degree to which ITEM has developed in various countries, the design and implementation problems, its positive and negative impact, and ways in which problems can be overcome. For that reason an approach for future research is proposed here.

\section{CHARACTERISTICS OF PAST RESEARCH}

\section{Research topics and methodology}

Visscher [2] presents a model which can be used to determine the features of ITEM-research as it has been carried out.

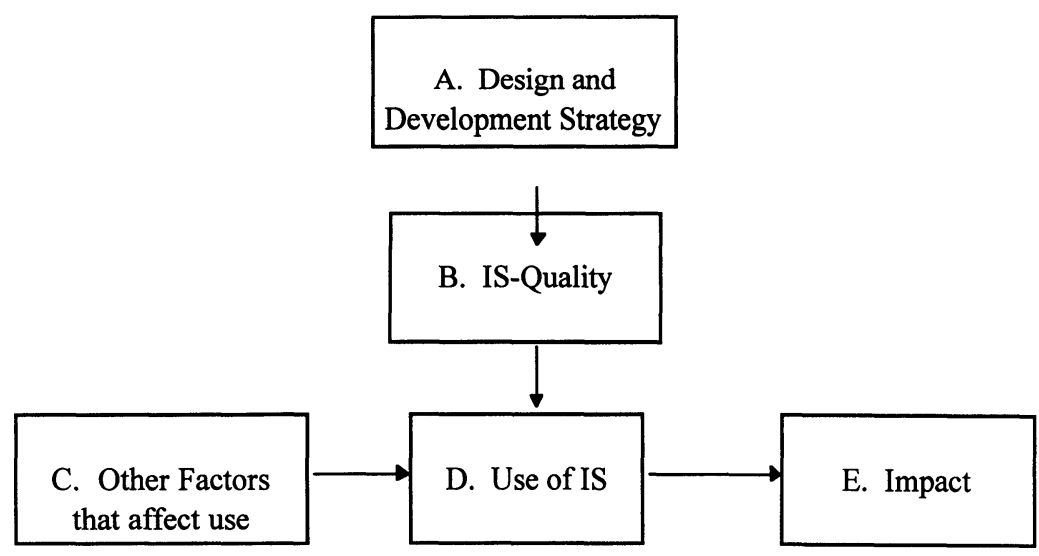

Fig. 1 Relationships between the design, use and effects of ISs 
The blocks in the model are interrelated: a choice in one block has consequences for what happens in one or more of the other blocks. Features of the design and development strategy used (block A) influence the quality of the resulting information system (block B) which probably has an impact on the magnitude of and the way of system usage (block D). Other factors (block C) affect system use as well as the nature of implementation processes in schools. Impacts of system use (block E) are supposed to be a function of the intensity with which, and the way in which an information system is used.

An overview of ITEM-studies can be obtained from two sources:

1. a special issue of the Journal of Research on Computing in Education on computer assisted school administration and management [3];

2. the proceedings of the Conference on Information Technology in Educational Management in Jerusalem [1].

These sources show ITEM-publications mainly to deal with:

- reflections/proposals regarding the way in which ISs should be designed and implemented, as well as possible ways of IS-usage and the expected (usually positive) impact of IS-use;

- descriptions of the characteristics of available computer applications;

- qualitative evaluations of experience gained with specific development and implementation strategies;

- empirical studies concerning the extent of system usage and features of user training;

- research on the positive and negative effects of school administrative computing.

So, most of the ITEM-literature deals with visions concerning the blocks in Figure 1, with descriptions of systems and strategies followed and, in a very small number of cases, with empirical studies concerning the implementation, use and effects of ISs. As a result clear insight into the relations (concerning various variables) between the different blocks in Figure 1 is missing.

As far as the research methodology is concerned ITEM-studies in most cases are one shot, small scale studies (mostly case studies). Subjective user perceptions are usually the basis for statements concerning system quality, usage and impact. More objective evaluations are rare. 


\section{RESEARCH NEEDED}

\section{Research topics and methodology}

Figure 1 suggests the following interesting research questions:

a) Which are the advantages and disadvantages of various strategies for information system design?

Designing ISs is a matter of making choices. Each design strategy most probably results in an IS with different characteristics (and therefore a different impact) and different user appreciation. The design approaches which result in the most appreciated and used ISs need to be studied, by carefully analysing strategy characteristics and by comparing resulting ISs.

b) Which characteristics do good ISs have, in terms of the kind of support they offer (e.g. information retrieval and document production, decision making support, decision making, communication) and how do they do this in terms of user friendliness and usability of information?

We know very little about the features of ISs which are appreciated and used intensely. We should analyze the features of such systems and find out why users value these systems so much.

c) Which implementation process features prove to be crucial for successful system implementation?

A high quality IS is no guarantee at all for its successful implementation [2]. Examples of factors which influence success are: the user attitude towards innovation, clarity of innovation goals and planning, available innovation resources, innovation encouragement by the principal and user training features.

This question should ideally be answered in longitudinal, large scale empirical research projects carried out after literature research and case studies have generated ideas about crucial implementation process variables.

d) In which way does the nature of educational organisations interact with IScharacteristics when implementing ISs?

Educational organizations are institutions with very specific features. Some features are general, like a high degree of teacher autonomy, limited policy making at school level and characteristic behaviour of school managers, but there are also organizational differences between schools (e.g. concerning their size, culture, policy making capacity).

The features of school organization potentially have implications for how an IS will be hosted. An IS therefore must embody assumptions about the nature of these organisations. The fit of the interface between an IS and the organizational context in which it is meant to be used, is crucial. We should 
find out which organizational features play a role and how we can take these into account as prerequisites for successful system implementation. The answer to this question should also be obtained by case study based large scale, longitudinal, empirical research.

e) At which levels and in which way are various computer applications used in educational organizations?

Just as designers choose to produce a system with specific characteristics, staff can decide to make use of an introduced IS in a certain way. The IS may, for instance, be used very intensely or very little, only for clerical purposes, or also to support decision making with respect to personnel, finance, the educational process and general pedagogical-didactical affairs. As mentioned before system usage is probably influenced by system quality and by features of implementation process and of school organization. Therefore, also the reasons for particular system usage should be studied: the causal relationship between block $\mathrm{B}$ and $\mathrm{C}$, and between block $\mathrm{B}$ and $\mathrm{D}$.

System usage until now has been studied very globally and only vague information on the intensity of system usage is available. We are in need of a diversity of objective in depth case studies of the actual use of Iss.

f) Which desired and undesired effects does system usage produce?

Most research makes little attempt to assess the effectiveness of the management information system under investigation, let alone relate findings about computer impact to overall effectiveness. Moreover, research on the organizational impact of system usage is seldomly carried out. And if any ISimpact is studied it is almost always at individual or at submit level. However, ISs are meant to improve organizational effectiveness by improving the information household, organizational coordination, control and problem solving, among others. An important evaluative question is therefore whether this desired increased effectiveness is actually realized. Also unplanned negative effects (e.g. increased monotonous data entry and retrieval work, work fragmentation, information overload) need to be studied. This question may be addressed by studying successful computer applications in relation with their overall effectiveness.

When studying the impact of a computer system, researchers should not base their findings on user perceptions, but make a comparison between a pretest (before IS introduction) and a posttest in relation to the original design criteria. Longitudinal projects are necessary to build up our knowledge in this respect. Visscher [4] reports an example of such a pretest posttest study on the effect on attendance of a computerized registration system. For measuring the effectiveness of all aspects of IS use a 'tool' will have to be developed. 


\section{g) How can identified problems be overcome?}

Answering the aforementioned six research questions will probably point to problems which need to be solved. Empirical studies on the questions $c$ to $g$ can give us information concerning factors influencing successful system design and implementation. On this basis experiments should be set up to gain experience with new design and implementation strategies and to investigate whether observed bottlenecks can be tackled in specific ways. An interesting experiment would for instance be to check whether new ways of user training taking account of previously identified problems, lead to more successful implementation.

\section{The user acceptance audit methodology}

So far, proposals for research have been formulated in general terms. A new interesting line of ITEM-research will be elaborated below which could contribute to the data collection.

Historically, education has not got a good record in evaluating change and the field of ITEM is no exception. The use of IT-systems in industry and commerce to support management processes has a longer history than that of the use in the educational system. This has given time for an awareness to develop about the importance of organizational needs, user needs and user acceptance at the implementation stage. This awareness developed from observing the problem of rejection or only partial success in about $80 \%$ of ITsystem implementations [5, 6]. Many system failure case studies which are relevant for the current situation in schools used acceptance audits to identify the problems.

The methodology of the User Acceptance Audit (UAA), developed to identify the source of problems within industry and commerce, can also be used within education to identify barriers and promoters to system success.

Wild et al. [7] report a pilot study on contextualizing the UAA for school based IT systems. The development of the UAA is described by Richardson [8] and Richardson and Otway [9]. Work so far carried out in contextualizing the UAA to evaluate IT systems in education is summarized in Wild [10]. The positive outcome from these pilot studies gives some confidence in the UAA methodology as a basis for evaluation of implementation and use of IT systems for school management.

The UAA focuses on the user, the task, context variables and the technology of the tool through the consideration of three core concepts:

- usability, the extent to which systems match user characteristics and the skills for the task concerned; 
- functional utility, how well a system fits the needs of a set of particular tasks;

- user acceptability, how willing users are to use the system in their own organization.

The criteria for acceptability fall into four further categories: ease of use, task match, user support and perceived consequences; the latter being used to assess a range of organizational and job related aspects which are affected by the computer system. Each UAA requires the creation of investigative tools based on these criteria, but tailored to be context specific using a combination of objective measures and subjective assessments. The objective measures require an examination of the physical environment, hardware configuration and general performance. Subjective assessments would normally be made using predefined questions for a semistructured interview.

Ease of Use covers the physical environment and the overall operating procedures of the system, such as navigation through the system, input procedures, screen layout and output procedures. In the pilot study, questions in this area highlighted some major problems in the error handling and printouts, both of which required manual alteration of records, and in some cases retyping of output data, before they could be released for management purposes. Problems with the environment, such as in the case where computer together with the operator is housed in a small 'cupboard' without windows or ventilation, can be identified by applying the requirements of the European Communities. Directive 90/270/EEC requires that workstations are evaluated on safety and health, that software is adaptable to the user's level of knowledge or experience and that systems display information in a format and a pace adapted to the operators.

Task Match is related to functional utility, but with particular emphasis on the tasks of the individual user. Ideally the study needs to form a continuum throughout design and implementation phases, but in reality evaluation is only done at a late stage of implementation. In practice it is found that the criteria of task match and ease of use overlap because a system which is matched to organisational needs, will be easier to use for specific required tasks. In the pilot study questions were considered in this area related to accuracy, relevance, completeness and timelyness of the data available from the system. Many problem areas were identified.

Support is of particular importance, especially at the start of implementation. Relevant questions relate to previous experience, initial training, local support and style, content and organization of user guides and manuals. Identification of learning difficulties and learning time for specific tasks provide useful 
information which can be used to direct future training. Users' reasons behind their answers in particular may shed light on the validity of assumptions made in providing support. It has been found that user guides are often at least one version behind the software and that these are indexed in terms of the software, rather than in terms of user tasks within the organization.

Perceived Consequences deal with user feeling about system use in terms of changed work pace, career prospects, job satisfaction, power and influence. And with how the IS has been integrated into the organizational structure. All these aspects relating to the 'job' influence user acceptance or rejection of the system.

In addition to the specific questions within the main criteria, a simple cost/benefit analysis can be used to quantify a 'general' feeling about the system. Further development of UAA involves a summary sheet of the findings which is presented during the semistructured interview and which allows users to prioritise issues across the criteria. This increases the UAA tool sensitivity in identifying issues of concern.

In most cases data is analyzed by simple frequency counts from responses on 1-5 scales and comments and discussion records are used to qualify the identified barriers and promoters of the system. The resulting information can be used for future training and system development.

\section{Further development of the evaluation methodology}

The pilot studies of the evaluation methodology have been carried out on financial and ordering systems of sixteen schools in the UK, and one particular management system. It is felt that the findings have illustrated the potential of the methodology. It should now be possible to extent the question base already available and do UAAs both on other ISs in the UK and on systems under development in other countries. However, as has been stressed before, it is important that the tool is contextualized for different systems which is best carried out via a pilot study in a small number of schools. The changing situation with respect to local educational management in many countries will also require changes to the question bank to ensure its relevance with respect to management decision making support.

The curriculum support provided by ISs is a problem for teachers are aware of the inadequacy of such systems. For example, they report that often transfer of data by hand, from a computer printout into a database or spreadsheet, is necessary for producing group lists in acceptable format. An analysis of the organization and ITEM implementation with respect to curriculum support is required. The 'context' will be wider than that needed for financial and ordering systems as all teachers are directly involved with the 
curriculum. Using sampling methods it should still be possible to provide an UAA which is both practical and valid.

\section{CONCLUSION}

The increasing importance of ITEM is in strong contrast with the scarcity of empirical research concerning the design and implementation of computerized systems for administrative and management support in education. Hopefully the directions for research proposed here will change this situation and enable educational institutions to benefit more from the support these systems can potentially provide.

\section{REFERENCES}

1. Barta, B.Z., Telem, M. \& Gev, Y. (1995) Information Technology in Educational Management. London: Chapman \& Hall.

2. Visscher, A.J. (1991) School Administrative Computing: a Framework for Analysis. Journal of Research on Computing in Education 24 (1) pp. 1-19.

3. Visscher, A.J., Spuck, D. and Bozeman, W. (1991) Computer Assisted School Administration and Management: An International Analysis. Special issue of the Journal of Research on Computing in Education, 24 (1).

4. Visscher, A.J. (1992) Design and evaluation of a computer-assisted management information system for secondary schools (Ph.D. thesis). Enschede: Universiteit Twente, Toegepaste Onderwijskunde.

5. Eason, K.D. (1988) Information Technology and Organisational Change. London: Taylor and Francis.

6. Plenart, G. (1988) The Basis of a Successful System. Information and Management 15, pp. 251-254.

7. Wild, P., Scivier, J.E. and Richardson, S.J. (1992) Evaluating Information Technology supported Local Management of Schools: The User Acceptability Audit. Educational Management and Administration 20 (1). 
8. Richardson, S. (1987) Operationalising usability and acceptability- $a$ methodological review, in Wilson, J.R., Corlett, E.N. and Manenica, I. (eds.), New methods in applied ergonomics, Taylor \& Francis.

9. Richardson, S. and Otway, H. (1985) User Acceptance of man-machine systems. Man-Machine Systems, Varse, Italy.

10. Wild, P. (1994) The use of task analysis and user acceptability audits in implementing information technology systems in schools, in Information Technology in Educational Management. London: Chapman \& Hall. 